\title{
Examining student ideas about energy measurements on quantum states across undergraduate and graduate levels
}

\author{
Gina Passante, Paul J. Emigh, and Peter S. Shaffer \\ Department of Physics, University of Washington, Seattle, Washington 98195, USA
}

(Received 29 September 2014; published 23 September 2015)

\begin{abstract}
[This paper is part of the Focused Collection on Upper Division Physics Courses.] Energy measurements play a fundamental role in the theory of quantum mechanics, yet there is evidence that the underlying concepts are difficult for many students, even after all undergraduate instruction. We present results from an investigation into student ability to determine the possible energies that can be measured for a given wave function and Hamiltonian, to determine the probabilities of each energy measurement and how they depend on time, and to recognize how a measurement of energy affects the state. By analyzing student responses to open-ended questions, we identify five broad, interrelated sets of conceptual and reasoning difficulties related to energy measurements. Data are drawn from sophomore-, junior-, and graduate-level quantum mechanics courses. Particular attention is paid to incorrect ideas that persist across all levels.
\end{abstract}

DOI: 10.1103/PhysRevSTPER.11.020111

PACS numbers: 01.40.Fk, 03.65.-w

\section{INTRODUCTION}

Experienced instructors recognize that quantum mechanics is a difficult subject. There is mounting evidence that many students complete a physics degree without having a firm grasp of some fundamental concepts [1-4]. Even majors that continue to graduate school in physics have similar difficulties [5-7]. However, a working knowledge of quantum mechanics is becoming increasingly important as the market for quantum technologies continues to grow. Thus, there is a need for ongoing research to document the problems that students encounter and to provide the kind of information needed to be able to develop and assess instructional strategies.

The energy eigenbasis defines the time dependence of the quantum state and is thus arguably the most important basis in quantum mechanics. Full understanding of a physical system is not complete without knowledge of the energy eigenvalues. In a typical course on quantum mechanics, students are asked to apply their knowledge of energy eigenvalues and eigenstates to answer questions about energy measurements. For example, they are often asked to determine the possible values that could result from an energy measurement and the probabilities of obtaining each value. Implicit in many questions is an understanding of the role of the Hamiltonian. Previous research studies have demonstrated that students struggle in answering these (and other) questions. The errors suggest a broad lack of understanding of the underlying quantum formalism that can

\footnotetext{
*Corresponding author. gpassante@fullerton.edu

Published by the American Physical Society under the terms of the Creative Commons Attribution 3.0 License. Further distribution of this work must maintain attribution to the author $(s)$ and the published article's title, journal citation, and DOI.
}

affect student ability to apply these principles to more complex and physically realistic contexts [8-14].

In this work we analyze student responses to a set of open-ended questions. The goal is to elicit common errors and probe the lines of reasoning that many students use. This detailed information not only informs the research base on student learning but can also guide the design of instructional materials. The results have implications for teaching both energy measurements and related topics, such as the WKB approximation, position and momentum measurements, and time evolution.

\section{CONTEXT FOR RESEARCH}

The research discussed in this paper has taken place in a sequence of courses at the University of Washington (UW). An undergraduate physics degree at UW requires between one and three courses on quantum mechanics. A sophomore-level course is the first formal introduction and is the only required course on quantum mechanics for physics minors and for one of the tracks for a physics major in our department. The course uses a spins-first approach that covers the first five chapters of McIntyre's textbook Quantum Mechanics [15]. It ends with solutions to the Schrödinger equation in position space for simple potentials. The second and third courses are at the junior level and together they cover the entire Griffiths' textbook Introduction to Quantum Mechanics [16]. Most of the data in this paper are drawn from these three courses. However, we also present data collected during the first week of instruction in a graduate course on quantum mechanics. These latter data are used to document the understanding of strong physics undergraduates after typical instruction on quantum mechanics.

In all cases, the data come from qualitative questions administered after relevant instruction. These questions 
have been designed to try to elicit explanations that provide insight into student conceptions and common chains of reasoning. Supporting evidence also comes from individual student interviews. Both the written questions and the interviews have probed student ideas related to energy measurements in a wide range of contexts from the infinite square well potential to perturbation theory.

The sophomore- and junior-level courses involved in this study have used preliminary versions of a set of tutorials for quantum mechanics that is being developed by our group [17]. Typically, the questions presented in this paper were asked before relevant tutorial instruction but after lecture instruction. However, in a few cases, they were asked after early versions of the tutorials that may have somewhat influenced the results. It is for this reason, in part, that we do not focus on the exact percentages of students who have specific difficulties. Percentages that are reported should be taken as a general measure of the prevalence of particular difficulties; the percentages obtained at other institutions may differ. However, the underlying difficulties reported in this paper have been present across all years of our investigation and have proved particularly resistant to instruction. Thus, we believe they are common among similar groups of students.

\section{RESEARCH PERSPECTIVE}

The perspective taken in this paper toward research on learning and teaching has grown out of the experience that the UW Physics Education Group has had in examining student understanding and developing research-validated curriculum. (See Ref. [18] for a more detailed discussion.) For this project, we began by trying to identify what students could and could not do through discussions with students in the classroom and analysis of written responses to questions on homework and course examinations. Our goal was to try to gain insight into the approaches that students use in solving a wide variety of problems. In the process we began to develop and modify questions that allowed us to probe incorrect lines of reasoning that seemed to be applied repeatedly by multiple students and that arose across a variety of questions and contexts.

The research involved members of our group independently interpreting many student statements as to the possible underlying approach taken by the students. Some responses did not include much detail, but were noted to be similar to more complete statements given by other students. In the process we came to identify certain approaches that we interpret as being consistent with one or more underlying conceptual or reasoning difficulties. We use the term difficulty to describe incorrect or inappropriate ideas or flawed patterns of reasoning that students use in answering one or more of the questions.

The difficulties that we discuss in this paper stood out during the analysis, as they occurred on a wide variety of questions in different contexts and often at several different levels of instruction. However, different formulations of each question often elicited different aspects of student thinking. Individual difficulties did not necessarily arise in equal percentages on all variations of the questions. Moreover, individual students did not always respond in the same way when asked different, related questions in the same course. The results suggest that students do not necessarily have a firmly held model that is driving their responses. Nonetheless, we are finding the identification of conceptual and reasoning difficulties to be fruitful in guiding the design of curriculum [17,19-21].

In this paper, we articulate five broad and overlapping sets of difficulties relating to energy measurements and discuss how these manifest at different levels of instruction. Those that are presented were chosen for several reasons: they are prevalent in different contexts (e.g., the infinite square well and perturbation theory), they occur at different levels of instruction (e.g., sophomore and junior), and they have been identified in every year of our study across multiple instructors. We include a discussion of some difficulties even if certain aspects have been documented previously in order to extend or contrast the results, or for completeness. Previous results from research are cited where appropriate.

\section{SAMPLE QUESTIONS FROM THE INVESTIGATION}

A functional understanding of energy measurements in quantum mechanics requires that students be able to interpret and apply steps in reasoning to contexts they have not previously encountered. In this study, we have focused on the ability of students to recognize and apply the following ideas: (1) a given quantum mechanical system has a set of allowed energy values and that these depend on the eigenvalues of the Hamiltonian, and thus, upon the potential, (2) a wave function defines the state of the system and provides information about the probability of measuring each energy, and (3) how time evolution and measurement can affect the possible measurement outcomes and the associated probabilities.

We have administered a wide variety of questions to probe student ideas about these aspects of energy measurements. In this paper, we illustrate three questions, each of which elicits student ideas about several ideas mentioned above. Multiple versions of each question have been asked in various quarters to ensure that the difficulties encountered are generalizable and not an artifact of the phrasing of the question or the context in which it is asked.

Question 1: Question 1, shown in Fig. 1, asks students to find the probability of an energy measurement at $t=0$ (part A) and $t>0$ (part B). Several versions have been given at the sophomore, junior, and graduate levels. The version in Fig. 1 was given on a final examination in a single sophomore quantum mechanics course at UW $(N=104)$. It indirectly probes student reasoning about 
Question 1: Recall that the eigenfunction of the infinite square well potential as given by $\phi_{n}(x)$. Collection $\mathrm{A}$ contains a large number of identical particles, each of which is described by the initial wave function

$$
\Psi_{A}(x, t=0)=\frac{1}{\sqrt{2}}\left(\phi_{1}(x)-\phi_{2}(x)\right) .
$$

A. Suppose you were to measure the energy of a very large number of particles at $t=0$. Would the number of particles with energy $E_{1}$ be greater than, less than, or approximately equal to the number of particles with energy $E_{2}$ ? Explain your reasoning.

B. At some later time, the wave function for the particles is given by

$$
\Psi_{A}(x, t)=\frac{1}{\sqrt{2}}\left(\phi_{1}(x) e^{-i E_{1} t / \hbar}-\phi_{2}(x) e^{-i E_{2} t / \hbar}\right) .
$$

Suppose you were to measure the energy of a very large number of particles at a later time. Is there a time $t>0$ when the number of particles with energy $E_{1}$ is greater than the number of particles with energy $E_{2}$ ? If so, determine at least one such time. If not, state why not.

FIG. 1. A sequence of questions given after all instruction in the sophomore quantum mechanics course. Similar questions have been given to students at the junior and graduate levels.

the probability of measuring various outcomes for the energy of a single quantum state by asking about a large number of measurements made on many identical systems.

A correct response to part A requires noting that the absolute square of the coefficients for both terms in the wave function are equal at the given time $(t=0)$. Thus, the number of particles measured to have energy $E_{1}$ is expected to be about the same as that for energy $E_{2}$. For part $\mathrm{B}$, in which the time dependence of the wave function is explicitly shown, the reasoning is similar. The time components vanish when the absolute square is taken, so there is no time when the number of particles measured to have energy $E_{1}$ is expected to be greater than the number measured to have energy $E_{2}$.

Versions of this question have been given to juniors and graduate students (see, for example, question 2A in Fig. 2), but usually with two differences. First, the questions have involved a single particle (rather than an ensemble) and students have been directly asked about the probabilities of various outcomes. Second, we have given students the state at an instant and have not provided the time dependence (as shown in part B of Fig. 1). This omission adds another step to the reasoning, since students need to identify the time dependence of each term in the wave function. These versions have been asked of more than 300 students at the junior level (after relevant lecture instruction) and of 31 students at the graduate level (before any graduate instruction).
Question 2: A particle in an infinite square well, with energy eigenstates $\Psi_{n}(x, 0)$, is prepared so that its wave function at time $t=0$ is:

$$
\Psi(x, 0)=0.6 \Psi_{1}(x, 0)+0.8 i \Psi_{2}(x, 0) .
$$

A. Suppose you were to measure the energy of this particle at time $t_{1}>0$. What value or values would a measurement of the energy yield?

B. Suppose that at a later time $t_{2}$ you measured the energy of the particle again. (Assume that the particle remains isolated from its surroundings during the time interval $t_{1}<t<t_{2}$.) Which of the following statements would best describe the result of your second energy measurement?

i) Definitely the same result as the first.

ii) Possibly the same result as the first.

iii) Definitely not the same result as the first.

C. A particle is prepared in the ground state of the infinite square well. Its position is then measured and found to be $x=L / 3$ (where $L$ is the length of the well). Suppose you now measured the energy of the particle. Which of the following statements best describes the result of your second energy measurement?

i) Definitely the ground state energy.

ii) Possibly the ground state energy.

iii) Definitely not the ground state energy.

FIG. 2. A sequence of questions that are given after approximately one month of instruction in the junior-level quantum mechanics course at UW. Students are also asked to explain their reasoning after each question.

Question 2: The question shown in Fig. 2 begins by providing students with a wave function at time $t=0$ written as a superposition of two energy eigenstates of the infinite square well. Variations of question 2 have been given to students in the junior-level course beginning in 1996.

Part A asks students to identify the values that could result from an energy measurement at time $t>0$. The correct answer is that both $E_{1}$ and $E_{2}$ are possible energy measurements since they correspond to the two eigenstates represented in the wave function. Part B asks students about possible results from an energy measurement made after the first energy measurement. To answer, students could recognize that after the first measurement the wave function of the particle corresponds to an energy eigenstate. Thus, a second measurement must yield the same result as the first.

In part $\mathrm{C}$, students are told that a position measurement has been made and are asked about the possible results of a subsequent energy measurement. A correct solution is to recognize that after the position measurement, the wave function is in an eigenstate of position (e.g., a delta function 
in position space), which can be written as a sum of many energy eigenstates. Therefore, a subsequent energy measurement could yield almost any energy eigenvalue for the potential. Question 2 has been given to more than 200 students at the junior level.

Note that, in this question, students are required to think about the state of a quantum system after a measurement has been made. In undergraduate and graduate textbooks, students are typically told that the possible outcomes of a measurement are the eigenvalues of the operator associated with the observable and that after a measurement the system is in an eigenstate corresponding to the observed eigenvalue (the "measurement postulate") [22]. Sometimes the phrase "collapse of the wave function" is used. There is some disagreement among instructors as to whether or not this postulate should be taught in undergraduate courses [27]. The arguments often focus on the difficulty (or, in some cases, impossibility) of performing such measurements. Nonetheless, many undergraduate courses discuss the state of a system after a measurement is made, and we have found that this context provides an excellent opportunity to probe student understanding of energy measurements.

Question 3: Question 3, shown in Fig. 3, involves a particle that is measured to be in the ground state of an infinite square well potential. At a later time, a perturbation is applied for a finite amount of time, after which it is removed. Students are told to treat the perturbation as beginning and ending instantaneously. They are asked to determine the values that could result from an energy measurement that is made (A) before, (B) during, or (C) after the perturbation is applied. Versions of this question have involved several different perturbations (e.g., a delta function or a small rectangular perturbation at the center of the well).

Question 3: A particle is measured to be in the ground state of the infinite square well at time $t<0$. At a later time, $t_{\mathrm{on}}>0$, a small perturbation is added to the center of the well. At a later time, $t_{\text {off }}$, the perturbation is removed.

A. Before the perturbation, $0<t<t_{\text {on }}$, what possible values could an energy measurement yield?

B. During the perturbation, $t_{\text {on }}<t<t_{\text {off }}$, what possible values could an energy measurement yield?

C. After the perturbation has been removed, $t_{\text {off }}<t$, what possible values could an energy measurement yield? Assume that no measurements where made while the perturbation was present.

FIG. 3. Question 3 has been given after lecture instruction on perturbation theory at the junior level. Questions testing similar ideas have been asked at different points in the junior year and at the graduate level.
Before the perturbation has been applied, only the ground state energy of the infinite square well can be measured, since the particle was initially in the corresponding eigenstate. For the instant at which the perturbation is applied, students should assume that the wave function is continuous. Therefore, the mathematical form just after the perturbation is applied still corresponds to that of the lowest energy eigenstate of the infinite square well. However, during the period that the perturbation exists, the new Hamiltonian determines the energy eigenstates of the wave function, and only the corresponding energies can be measured. At the start of the perturbation, the wave function in general will not be in an eigenstate of the new potential and its time evolution is determined by the new energies. Thus, the wave function will evolve out of the initial unperturbed eigenstate. Finally, at the instant the perturbation is removed, the wave function can again be assumed to be continuous. The energies that can be measured after the perturbation is removed are once again those corresponding to the square well. However, at this point the wave function corresponds to a superposition of many energy eigenstates of the infinite square well and a measurement of the energy is no longer restricted to the ground state energy. More than 140 students at the junior level have answered versions of this question. The results are similar when it is given before or after lecture instruction on time-dependent perturbation theory.

\section{STUDENT UNDERSTANDING OF ENERGY MEASUREMENTS}

In responding to the questions described in the previous section, students have made many, various errors. We have identified five broad categories of conceptual and reasoning difficulties that appear to underlie many of their answers and that seem to persist throughout instruction. In this section we provide examples of student responses when they help to illustrate or substantiate specific difficulties. It is important to recognize that individual student statements may fit into several categories and that no single statement completely encapsulates a particular difficulty. The categories presented have been developed on the basis of examining a large number of student responses to multiple different questions and draw on data obtained from individual student interviews. Less formal discussions with students in class or in office hours have also contributed to the analysis. The percentage of students who made a particular error is included for those cases in which the responses did not appear to depend strongly on the version of the question that was asked.

\section{A. Failure to understand the relationship between the wave function and possible energy measurements}

In their responses to the questions above, many students revealed a lack of understanding of the relationship 
between the wave function and the possible energy values and their corresponding probabilities. Some of the questions probed student ability to find these quantities from the wave function, others examined student ability to reason in reverse (e.g., by asking students to find the possible results of an energy measurement after a first measurement has been made). The latter reasoning is arguably more difficult, since students must first determine the wave function after an energy measurement and then use it to obtain information about a future measurement.

\section{Finding the probability of an energy measurement from the wave function}

Research by others has demonstrated that students often think that an energy measurement can yield the expectation value of energy [9] or they calculate the probability density in order to find the probability of an energy measurement [8]. In our research we have also identified these errors at the junior level; however, we find that these errors are less prevalent among graduate students, and they did not appear at all in sophomore students responses to question 1.

The errors made by students in the sophomore-level course often seemed to be at a more basic level than those mentioned above. A common error was to relate the probability of making a specific energy measurement directly to the coefficient of the corresponding term in the wave function-without performing the absolute square $(15 \%, N=104)$. Some students did this explicitly. For others this error was more implicit. See, for example, the following response to question 1B (Fig. 1):

These [energy eigenstates] are rotating around according to the Schrödinger time evolution as given by $e^{i E_{n} t / \hbar}$. The value for $i E_{n} / \hbar$ is not the same so they are rotating at different speeds so at some point the probability of being in $E_{1}$ will be greater than $E_{2}$. [sophomore, question $1 B]$

This student appears to have a good understanding of the time evolution of this wave function. He describes the rotation of the real and imaginary components of the wave function in the complex plane. However, the student does not correctly associate the square of the coefficients in the wave function to the probabilities of the energy measurements. Like many of the students on this question, he seems to be treating the real part of the coefficient as the only important component.

The tendency to focus on the real part of the wave function was not limited to students in the sophomore course. One of the variations of question 1B asked in the junior-level course presented students with the wave function $\Psi(x, 0)=i \sqrt{\frac{1}{3}} \psi_{0}-\sqrt{\frac{2}{3}} \psi_{1}$ and asked whether there is a time when the probability of measuring $E_{0}$ is equal to 1 :
Yes, $\psi_{1}$ also rotates through the complex axis in time, meaning there are times at which it is completely imaginary, and thus unobservable. [junior, variation of question 1B]

This student explicitly states that only real parts of the wave function can be measured. Approximately 10\% $(N=123)$ of the junior-level students gave answers consistent with treating an imaginary coefficient for a term in the wave function as meaning that the corresponding energy value is not measurable.

Some of the students who did not properly square the coefficient also treated the complex exponential as if it were instead a decaying exponential. This tendency is discussed in greater detail in Sec. V D.

Even some students at the graduate level struggled with versions of question 1B. For example, when asked if the probability of measuring a particular energy at a later time is the same as it is at $t=0$, many students explained that the time dependence of energy measurements depends on the time dependence of the state. Statements like the following were common:

It [the probability of measuring $E_{n}$ at a later time] should be the same [at all times]. $|\psi\rangle$ is not relevant with t. So $\langle\psi|\hat{H}| \psi\rangle$ doesn't change. [graduate, variation of question 1B]

This student obtained the correct answer, but the underlying reasoning is flawed, since $|\psi\rangle$ does in fact depend on time [28].

\section{Determining the outcomes of a subsequent energy measurement}

Many of the responses to the questions suggested that students were having difficulty in determining the wave function after an energy measurement is made. To answer questions $2 \mathrm{~B}$ and $3 \mathrm{~A}$, for example, students must recognize that after the energy measurement, the wave function corresponds to an energy eigenstate. Thus, there is only one possible outcome for a future energy measurement. Although these questions do not explicitly ask students to write the wave function after the first measurement, the explanations indicate that many failed to recognize or apply this key idea. On question 2B, for example, $45 \%$ of juniorlevel students $(N>200)$ incorrectly answered that the result of a second measurement would possibly, but not necessarily, be the same as the first. The following student statement is exemplary:

The particle is described by a wave function with elements in both eigenstates. Although a measurement of energy collapses it to one, the possibility of the other still exists, so a second measurement could get the other E. [junior, question 2B] 
This student states that there is still a nonzero probability associated with the other energy value, even though he has knowledge of a "collapse" of the wave function. These results corroborate findings by Zhu and Singh [8].

We have asked questions similar to the one above but differ in that students are not given the initial wave function, but rather are told the result of the first energy measurement, e.g., that the measurement yielded the ground state energy. (See, for example, question 3A.) Lacking information about the state before the measurement, students often answered that any of the energy eigenvalues of the Hamiltonian could be obtained.

The failure to recognize how a measurement affects the state of a system does not seem to be limited to measurements of energy. In the sophomore course, we have asked similar questions about measurements of spin. Many of the same types of difficulties have emerged.

\section{B. Tendency to associate the time dependence of energy measurements with properties of stationary states}

Some previous studies have demonstrated that students often have difficulty in identifying stationary states or in answering questions that ask explicitly about properties of stationary states [8]. We have found that even on questions that do not involve stationary states, students spontaneously invoke the term, often incorrectly, in justifying their ideas about how the energy of a state does or does not change with time. [In response to question 1B, about $10 \%$ of the sophomore students $(N=104)$ explained their answer in terms of stationary states.]

Stationary states are energy eigenstates and as such their probability densities do not change with time. We have found that students at all levels have a tendency to extend this property both to the wave function and to the probability of energy measurements. For the scope of this paper, we restrict ourselves to discussing the latter. (See our companion paper for a discussion of the former [29].)

Consider the following explanation of question $1 \mathrm{~B}$ regarding the state $\Psi_{A}(x, t)=\frac{1}{\sqrt{2}}\left[\phi_{1}(x) e^{-i E_{1} t / \hbar}-\phi_{2}(x) e^{-i E_{2} t / \hbar}\right]$ :

$\Psi_{A}(x, t)$ is a stationary state, the probabilities [of energy measurements] will not change. [sophomore, question 1B]

The student has provided the correct answer, but has made two important errors. First, this student has incorrectly identified the wave function as being a stationary state [30]. Second, this student has indicated that because it is a stationary state the probability of energy measurement outcomes will not change in time. Although the probabilities do not depend on time, this is true for all quantum states in a time-independent potential, not just stationary states.
A similar question was asked on a final examination in the junior-level course. Students were given a three-dimensional wave function $\psi(r, \theta, \phi, t=0)=$ $N\left[\psi_{321}+2 \psi_{311}+2 \psi_{210}\right]$, where $\psi_{n l m}$ are the normalized energy eigenfunctions with energy eigenvalues $E_{n l}$. They were asked to find the probability that an energy measurement at $t=0$ would result in $E_{21}$ and asked whether or not their answer would change if instead the energy were measured at a later time $t>0$. Almost all students (approximately $80 \%, N=44$ ) correctly answered that the probability would not change if it were measured at a later time; however, only $25 \%$ gave correct explanations. Many reasoned using stationary states, as demonstrated as follows:

No because all [three terms] are energy eigenstates which do not change with time. They are stationary. [junior, variation of question 1B]

It [the probability of an energy measurement] would not change. The eigenvectors of energy are stationary states, so their coefficients will not be changing in time. [junior, variation of question 1B]

The first student correctly identifies the $\psi_{\mathrm{nlm}}$ as stationary states, but then states, incorrectly, that they do not depend on time. The student then uses that as the basis for stating that the energy probabilities do not depend on time. The second student also gives a correct answer, but explicitly states that since each term in the wave function corresponds to a stationary state, none of the coefficients have a timedependent phase. Although each of these students arrives at the correct answer, they do not correctly relate the time dependence of quantum states to the time independence of the probability of energy measurements. We have found that some graduate students make similar errors, although less frequently.

\section{Difficulties related to outside knowledge}

On all of the questions we have asked, some students have answered based on ideas that lie outside of the model for quantum mechanics that has been developed in class. The specific errors and the types of reasoning have varied depending on the level of instruction.

On question 1 (Fig. 1), a consistent fraction of the students (about 10\%, $N=104$ ) in the sophomore course stated that lower energy values are more likely to be measured than higher values. (Recall that the particle is in an equal superposition of two energy eigenstates.)

[The probability of finding the particle in the ground state is] greater than [the first excited state]. It requires more energy for particles to stay in $E_{2}$ than $E_{1}$. So particles are more likely to be in a more stable state $E_{1}$. [sophomore, question 1A] 
This student does not seem to recognize that the probabilities for the energy measurements are determined by the corresponding coefficients in the wave function. (See the discussion in Sec. VA.) A possible explanation for this response is that the student is using reasoning from classical physics about particles being more likely to have the lowest available energy. Another explanation is that the student recalls information about spontaneous emission. In either case, the student is neglecting to apply the model that has been developed in class.

We found that reasoning based on outside knowledge was more prevalent at the junior level when the questions involved subsequent measurements. For example, on question 2B (Fig. 2), about 10\% $(N=141)$ of students used conservation of energy to explain the possible outcomes of future energy measurements. The following response is typical of these students:

Since no energy was added or subtracted [during the measurement], there MUST be the same $E$ due to the law of conservation of energy. [junior, question 2B]

This student arrived at the correct answer, but did so by assuming that the prior measurement did not affect the energy of the system. The same reasoning yields an incorrect answer when it is applied to energy measurements performed after a position measurement (or a measurement of any other incompatible observable). For example, on question $2 \mathrm{C}$, some students stated that the position measurement did not add energy to the system, so the energy distribution will be the same as before the position measurement. Other students, like the one following, stated that the position measurement would add energy to the system, so a subsequent energy measurement would result in an increased value:

The particle's position was measured which means some energy must have been [added] into the system-which would excited the particle above the ground state. [junior, question 2C]

We have also seen instances of this chain of reasoning from graduate students. The explanation below was given in response to a question similar to question 3 (Fig. 3). In this version, the potential was instantly changed from the infinite square well to the simple harmonic oscillator and students were asked about the possible results of an energy measurement some time after the change:

The energy will be unchanged. I'm guessing this because I don't want to violate conservation of energy. [graduate, variation of question 3B]

Some students used similar reasoning in their responses to question $3 \mathrm{~B}$, in which a small perturbation is added to an infinite square well. In many versions of the question, the perturbation is nonzero over only a small portion of the well (e.g., a small constant positive or negative correction applied to a finite region near the center of the well). Some students reasoned that the possible energy measurements are found by adding the value of the perturbation to the energy eigenvalues for the infinite well. In some cases, students thought that the energy that could be measured would depend on where inside the well it was measured. For example, the possible energies would be those of the unperturbed infinite square well if the measurement were made where the perturbation is zero.

Related to these errors is a tendency of students to treat the energy of any system as being well defined (i.e., there is only one possible outcome from an energy measurement). Perhaps students who indicate that the expectation value of the energy could result from a single energy measurement (see Sec. VA) are using this reasoning. Interactions with students during tutorials and office hours provide us with additional support that this may be affecting some of their answers.

\section{Failure to recognize that the time evolution of an isolated system is determined by the Schrödinger equation}

On some of the questions that we have asked, student responses have reflected a tendency to ascribe a time dependence to isolated systems that goes beyond that described by the Schrödinger equation. Students have often predicted that a particle will decay to the ground state, return to its initial state (revival), or evolve to include all possible values (diffusion) [31]. In some cases, students are using knowledge from outside the course, as was discussed in the previous section. The difficulties below are treated separately since they all seem to be related to the dynamics of quantum systems.

Decay reasoning.-In some versions of the questions that we have administered, students are asked about the possible energies that can be measured shortly after an energy measurement and a long time afterward. On these versions, many students respond that the particle will eventually decay into the ground state. We also see evidence of this in the time-dependent perturbation theory question in Fig. 3, for which some students have stated that the particle will eventually end up in the ground state. This reasoning is most common when students are told that the particle begins in an excited state (as opposed to the ground state):

As time goes on the particle is more likely to settle into the ground state whatever that may be. [junior, question 3B]

Many students fail to provide a detailed explanation for why they think the particle will evolve to be in the ground state. However, the reasoning given by some suggests that they are recalling ideas they had studied 
earlier, such as radioactive decay and spontaneous or stimulated emission.

Revival reasoning.-On questions that ask about the possible energy values that could be measured after a prior energy measurement, students often indicate that the particle will return to the initial state. Others do not explicitly indicate that it will return to that state, but they give probabilities for measuring the energy that are the same as they were before the measurement. These types of answers were given by about $20 \%(N=141)$ of the students on question 2B (Fig. 2). A sample response is as follows:

The time interval, minute, is long enough for the wave to return back to normal, so the option for both [energy measurements] is there like before. [junior, question 2B]

It is not clear what this student means by the term normal; however, the student clearly states that both measurement options are possible, even after the first energy measurement has been made.

We also have found that many students express the idea that a quantum system has a "memory" in time-dependent perturbation theory. For example, in question 3C (Fig. 3) students often predict that the particle will return to its initial state once the perturbation is removed. This idea is not restricted to the context of energy measurements. On questions involving successive spin measurements, students often express similar ideas.

Diffusion reasoning.- - In response to many of the questions we have asked, some students have responded that after a measurement has been made, the state will eventually consist of an equal superposition of all available eigenstates. The following student statement is in response to a version of question 2B (Fig. 2) in which a particle was first measured to have energy $E_{1}$ and the question asks what are the possible results of a subsequent energy measurement:

If the energy were measured immediately afterward, the probability is likely to remain $E_{1}$ because the wave function has not yet spread out into its full linear combination. However, if the energy is measured a long time later, then the probability of measuring $E_{1}$ decreases, because the particle is now in a superposition of states whose energies are clearly not all $E_{1}$. [junior, variation of question $2 B$ ]

We have also observed this line of reasoning in other contexts, such as angular momentum. For example, if a particle is known to have the angular momentum quantum numbers $l=1$ and $m_{l}=1$ (where $m_{l}$ is the quantum number for the $z$ component of angular momentum), then the possible quantum numbers for angular momentum in the $x$ direction are $-1,0$, and 1 . Many students incorrectly state that each option must have an equal probability of $1 / 3$.

\section{E. Failure to recognize the role of the Hamiltonian in determining the possible energy values}

We have found that many students have difficulty in relating the Hamiltonian to the possible energy values for a system. Two types of errors were common in response to the questions we asked.

The first type of error was evident on versions of question $1 \mathrm{~B}$, all of which involve a superposition of two energy eigenstates. The questions probe student understanding that the probability of energy measurements for such a system does not change in time. Many students answered correctly and correctly stated that the absolute squares of the coefficients are not time dependent. However, some based their reasoning on the fact that the potential is not changing [32]. These responses are consistent with the belief that the probabilities do not have time dependence if the Hamiltonian is time independent. We have also paired this question with one that asks about the time dependence of the wave function, and have found that students have answered in the same way for both questions. This suggests that the difficulty is more general and reflects a misunderstanding of the role of the Hamiltonian in quantum systems [33].

The second type of error was demonstrated in responses to question 3 (Fig. 3), which asks about possible energy measurements for a particle in the ground state of a system, before, during, and after a perturbation. Almost 30\% ( $N=140$ ) of the (junior-level) students incorrectly indicated that the original, infinite square well energies could be measured while the perturbation is present. These students seem to be using the basis in which the wave function is written, and not that for the current Hamiltonian, to determine the possible results of an energy measurement. This error also occurred for the period after the perturbation has been removed. About $40 \%$ answered correctly that any of the energy values associated with the original, unperturbed potential could be measured; however, about half of them have stated that it takes time for the particle to "feel the effects" of the change to the potential. They do not seem to be arguing that the potential itself takes time to change, but rather that the wave function needs time to adapt. The explanations suggest that students are treating the wave function, and not the Hamiltonian, as determining the possible outcomes of an energy measurement [34].

Graduate students gave very similar responses on a variation of question 3 . This version began with a particle in the first excited energy eigenstate of an infinite square well. Students were told that the potential then instantaneously changes to that for a harmonic oscillator and were asked to list the possible results of an energy measurement made immediately after the change. Only $25 \%(N=31)$ of the graduate students answered correctly. About 40\% incorrectly stated that the original infinite square well energy could still be measured, as follows: 
It should still be $E_{2}$ - the wave will take time to conform. [graduate, variation of question $3 B$ ]

Many, like the student above, reasoned that it would take time for the particle to feel the effects of the change. About half of the students who gave this response predicted that some time later the harmonic oscillator energies would be measured.

\section{DISCUSSION}

In this paper, we have documented some of the difficulties that students have in reasoning about the results of energy measurements on quantum systems. We have probed student thinking about which energy values can be measured for a given physical system, how to calculate the probabilities of obtaining each energy value, and how both time evolution and measurement can affect these probabilities. The results provide insight into some of the conceptual and reasoning difficulties that students encounter in the study of quantum mechanics. We have found it useful to group the underlying problems into five related categories. These are not limited to sophomore- or juniorlevel students, most also arise at the graduate level.

At the sophomore level, many of the errors seem to be relatively basic in nature, such as a failure to use the absolute square of the coefficients in a wave function to find the probabilities for the measurements of energy. However, the errors often seemed to have conceptual underpinnings. For example, some responses are consistent with a belief that only the real part of the wave function contributes to the energies that can be measured and their associated probabilities. This and other ideas seem to persist to the junior level and beyond. In the sophomorelevel course, for example, we found evidence of students treating the term stationary state as implying that the wave function itself (not just the probability density) has no time dependence. The students then used this reasoning as the basis for arguing that the probability of energy measurements do not depend on time. This incorrect chain of reasoning proved to be persistent, and we saw evidence for it at both the junior and graduate levels.

One of the most interesting errors we found at the sophomore level was evident in only a small fraction of the students $(\sim 5 \%)$, but the reasoning they used provided insight into errors made by students at more advanced levels. Some of the sophomores seemed to be reasoning that the probability of a given energy measurement depends on time only if the potential has a time dependence. Although this chain of reasoning was not common among sophomores, it was relatively common at the junior level in the context of perturbation theory. We had seen this reasoning previously among these students and thought it was an isolated and question-dependent error. However, the underlying ideas appear to be much deeper, and suggest that many students fail to understand the relationship between the time-dependent and time-independent Schrödinger equations.

At the junior level we also asked questions about subsequent measurements made on a system. The results suggest that there is considerable confusion about the effect of a first measurement on the possible outcomes of later energy measurements. Students often gave answers that suggested they thought that the particle eventually returns to the initial state or would evolve to include all possible energies. Some provided explanations that indicated that the energy of a system does not change when a measurement is made.

Finally, at both the junior and graduate levels we found that when faced with an abrupt change to the potential, a large fraction of students claim that the energy eigenvalues of the former potential can still be measured after the change. They reason it would "take time for the particle to feel the effects of the change." These students seem to be aware that the potential determines the possible energy values, as many of them articulate that, some time after the change, the energy eigenvalues of the new potential can be measured. However, instead of using knowledge of the instant the measurement is made, they appear to be incorporating their knowledge of the system moments earlier.

We have found that a critical step in the design of effective curriculum is the identification of ideas that underlie the errors that students make on a given topic. This process is guiding the design of curriculum for the junior-level quantum course (Tutorials in Physics: Quantum Mechanics [17]). At this stage, there is evidence that some of these tutorials have a direct impact on student conceptual understanding [35]. However, significant work still needs to be done. Some of the most common difficulties seem to be persistent and resurface when students are asked questions in different ways or in new, more difficult contexts. The discussion in this paper has focused on some of the areas that we have found relevant for most topics in quantum mechanics, and in recent versions of the tutorials we have made an effort to continually reinforce many ideas relating to energy measurements throughout the undergraduate course content.

\section{ACKNOWLEDGMENTS}

The authors would like to thank past and present members of the Physics Education Group at the University of Washington. In particular, we would like to acknowledge Bradley Ambrose and Andrew Crouse for their research efforts that helped to start the quantum mechanics effort at UW. We would also like to thank the faculty who welcomed this research into their classrooms: John Rehr, Andreas Karch, Blayne Heckel, and Daryl Pedigo. This research has been funded in part by the National Science Foundation under Grant No. DUE1022449. 
[1] A. Crouse, Ph.D. thesis, University of Washington, 2007.

[2] D. F. Styer, Common misconceptions regarding quantum mechanics, Am. J. Phys. 64, 31 (1996).

[3] C. Singh, Student understanding of quantum mechanics, Am. J. Phys. 69, 885 (2001).

[4] I. D. Johnston, K. Crawford, and P. R. Fletcher, Student difficulties in learning quantum mechanics, Int. J. Sci. Educ. 20, 427 (1998).

[5] C. Singh, Student understanding of quantum mechanics at the beginning of graduate instruction, Am. J. Phys. 76, 277 (2008).

[6] E. Cataloglu and R. W. Robinett, Testing the development of student conceptual and visualization understanding in quantum mechanics through the undergraduate career, Am. J. Phys. 70, 238 (2002).

[7] L. D. Carr and S.B. McKagan, Graduate quantum mechanics reform, Am. J. Phys. 77, 308 (2009).

[8] G. Zhu and C. Singh, Improving students' understanding of quantum measurement. I. Investigation of difficulties, Phys. Rev. ST Phys. Educ. Res. 8, 010117 (2012).

[9] G. Zhu and C. Singh, Students' difficulties with quantum measurement, AIP Conf. Proc. 1413, 387 (2012).

[10] G. Zhu and C. Singh, Surveying students' understanding of quantum mechanics in one spatial dimension, Am. J. Phys. 80, 252 (2012).

[11] E. Gire and C. A. Manogue, Making sense of quantum operators, eigenstates and quantum measurements, AIP Conf. Proc. 1413, 195 (2012).

[12] E. Gire and C. A. Manogue, Resources students use to understand quantum mechanical operators, AIP Conf. Proc. 1064, 115 (2008).

[13] S. Goldhaber, S. J. Pollock, M. Dubson, P. Beale, and K. K. Perkins, Transforming upper-division quantum mechanics: Learning goals and assessment, AIP Conf. Proc. 1179, 145 (2009).

[14] C. Singh, Assessing and improving student understanding of quantum mechanics, AIP Conf. Proc. 818, 69 (2006).

[15] D. H. McIntyre, Quantum Mechanics (Pearson Education, San Francisco, 2012).

[16] D. J. Griffiths, Introduction to Quantum Mechanics, 2nd ed. (Pearson/Prentice-Hall, Upper Saddle River, NJ, 2005).

[17] L. C. McDermott, P. S. Shaffer, P. R. L. Heron, and the UW Physics Education Group, Tutorials in Physics: Quantum Mechanics, preliminary edition ed. (2014).

[18] P. R. L. Heron, Empirical investigations of student understanding, AIP Conf. Proc. 720, 15 (2004).

[19] L. C. McDermott and P. S. Shaffer, Research as a guide for curriculum development: An example from introductory electricity. Part I: Investigation of student understanding, Am. J. Phys. 60, 994 (1992).

[20] P. S. Shaffer and L. C. McDermott, Research as a guide for curriculum development: An example from introductory electricity. Part II: Design of instructional strategies, Am. J. Phys. 60, 1003 (1992).

[21] P. R. L. Heron, in Empirical investigations of learning and teaching. Part I: Examining and interpreting student thinking, in Proceedings of the International School of Physics "Enrico Fermi," Course CLVI, edited by E. F. Redish and M. Vicentini (IOS Press, Amsterdam, 2004), pp. 341-350.

[22] All of the commonly used textbooks that we reviewed discuss measurements in this way. See, for example, Refs. [15,16,23-26].

[23] R. L. Liboff, Introductory Quantum Mechanics (AddisonWesley, Reading, MA, 1980).

[24] N. Zettili, Quantum Mechanics: Concepts and Applications (John Wiley \& Sons, Chichester, England, 2001).

[25] J. J. Sakurai, Modern Quantum Mechanics, revised edition (Addison-Wesley, Reading, MA, 1994).

[26] R. Shankar, Principles of Quantum Mechanics, 2nd ed. (Plenum Press, New York, 1994).

[27] M. Dubson, S. Goldhaber, S. J. Pollock, and K. K. Perkins, Faculty disagreement about the teaching of quantum mechanics, AIP Conf. Proc. 1179, 137 (2009).

[28] He also seems to treat $\langle\psi|H| \psi\rangle$ as representing the probability of an energy measurement rather than the expectation value. Similar tendencies have been reported in Ref. [8].

[29] P. J. Emigh, G. Passante, and P. S. Shaffer, Student understanding of time dependence in quantum mechanics, Phys. Rev. ST Phys. Educ. Res. 11, 020112 (2015).

[30] In our study, we found that many students treated the sum of stationary states as also being a stationary state. This error is common and has been documented in several prior studies.

[31] These have all been previously identified in Ref. [1], and the revival evolution has also been discussed in Refs. $[8,10]$.

[32] It is correct in that, if the potential did depend on time, then the probability of the energy measurements would change. However, in that case, the measurable energy values themselves would also vary.

[33] One question of the Quantum Mechanics Survey (QMS) asks students directly if the Hamiltonian determines the time evolution of a quantum system [10].

[34] A question on the QMS involves the reverse line of reasoning. Students are asked whether or not the Hamiltonian predicts the form of the stationary state wave functions [10].

[35] G. Passante, P. J. Emigh, and P. S. Shaffer, in Proceedings of the 2014 Physics Education Research Conference, Minneapolis, MS, 2014, edited by P. V. Englehardt, A. D. Churukian, and D. L. Jones (2015), www .compadre.org/per/perc/conference.cfm?Y=2014\#PRP46. 